\title{
Management of Patients Presenting with Acute Subdural Hematoma due to Ruptured Intracranial Aneurysm
}

\author{
Serge Marbacher, Ottavio Tomasi, and Javier Fandino \\ Department of Neurosurgery, Kantonsspital Aarau, 5001 Aarau, Switzerland \\ Correspondence should be addressed to Serge Marbacher, serge.marbacher@ksa.ch \\ Received 12 September 2011; Revised 14 November 2011; Accepted 28 November 2011 \\ Academic Editor: Mark Morasch
}

Copyright ( $) 2012$ Serge Marbacher et al. This is an open access article distributed under the Creative Commons Attribution License, which permits unrestricted use, distribution, and reproduction in any medium, provided the original work is properly cited.

\begin{abstract}
Acute subdural hematoma is a rare presentation of ruptured aneurysms. The rarity of the disease makes it difficult to establish reliable clinical guidelines. Many patients present comatose and differential diagnosis is complicated due to aneurysm rupture results in or mimics traumatic brain injury. Fast decision-making is required to treat this life-threatening condition. Determining initial diagnostic studies, as well as making treatment decisions, can be complicated by rapid deterioration of the patient, and the mixture of symptoms due to the subarachnoid hemorrhage or mass effect of the hematoma. This paper reviews initial clinical and radiological findings, diagnostic approaches, treatment modalities, and outcome of patients presenting with aneurysmal subarachnoid hemorrhage complicated by acute subdural hematoma. Clinical strategies used by several authors over the past 20 years are discussed and summarized in a proposed treatment flowchart.
\end{abstract}

\section{Introduction}

Rupture of a cerebral aneurysm normally results in subarachnoid hemorrhage (SAH) and is often complicated by intracerebral hematoma (ICH), but only on rare occasions does it cause acute subdural hematoma $(\mathrm{aSDH})$ [1]. Diagnosis of aneurysmal SAH can be difficult in comatose patients in whom loss of conscious due to aneurysm rupture results in or mimics a traumatic brain injury [2]. Determining a differential diagnosis and treatment modalities can further be complicated by the rapid clinical course and the mixture of symptoms due to the ruptured aneurysm and the mass effect of the hematoma.

Rapid decision making is required to treat this lifethreatening condition. The majority of patients with aneurysmal SAH and coincidental acute subdural bleeding present in a severe clinical condition, and immediate surgical management is required [2-4]. Decisions to be made include whether preoperative diagnostic studies should precede surgery and whether obliteration of the aneurysm should be performed during hematoma evacuation or in a separate delayed intervention after resuscitation procedures.

The incidence of combined SAH and aSDH varies from $0.5 \%[5,6]$ to $10 \%[7]$ in clinical series. The rarity of aneurysmal aSDH makes it difficult to design reliable clinical treatment guidelines. Large systematic series do not exist, and thus treatment decisions are mainly based on personal experience. The aim of this review is to propose a management flow chart and protocol based on published experience with such cases over the past two decades.

\section{Materials and Methods}

2.1. Search Strategy. The literature was screened for case studies of acute subdural hematoma secondary to ruptured intracranial aneurysm. Articles for this review were identified by MEDLINE PubMed database searches of the literature from January 1990 through December 2009 using the terms "acute subdural hematoma," "subarachnoid hemorrhage," and "cerebral aneurysm" (by using the Boolean operator AND) (Table 1). The senior author independently assessed the reproducibility of the search strategy on August 30, 2010, two days after the first author's search. Cross-references were checked in each eligible article.

2.2. Selection Criteria. Articles were excluded based on title and abstract because they (i) were not written in the English language, (ii) were technical notes or laboratory 
TABLE 1: Search Strategy*.

\begin{tabular}{llc}
\hline Search number & $\begin{array}{l}\text { Process description } \\
\text { ("key words") }\end{array}$ & $\begin{array}{c}\text { Results } \\
\text { (no. of articles) }\end{array}$ \\
\hline no. 1 & Search “cerebral aneurysm" & $\mathbf{2 2 9 4 4}$ \\
no. 2 & Search “subarachnoid hemorrhage” & $\mathbf{1 7 8 8 3}$ \\
no. 3 & Search “subdural hematoma” & $\mathbf{7 7 3 2}$ \\
no. 4 & Search \#1 AND \#2 AND \#3 & $\mathbf{1 5 5}$ \\
no. 5 & Search “01/1990-12/2009” AND \#4 & $\mathbf{8 5}$ \\
\hline
\end{tabular}

*All searches for this study were performed on August 28, 2010, by the first author and verified by the second author on August 30, 2010. The publication date limits were set to January 1990-December 2009.

investigations, or (iii) were not peer-reviewed/original studies. The remaining articles were selected for inclusion if the patients were adults and the single cases or case series provided detailed descriptions of clinical characteristics and patient management.

2.3. Data Acquisition. From selected cases, we extracted the following characteristics and recorded them in a data sheet: age; gender; initial clinical findings, including Glasgow Coma Scale (GCS) [8] score, clinical SAH grade based on the Hunt and Hess $(\mathrm{H} \& \mathrm{H})[9,10]$, and the World Federation of Neurological Surgeons (WFNS) [11] classifications; presence of major (aphasia, hemiparesis, or hemiplegia) and minor (cranial nerve palsies) focal neurological deficits, hemodynamic situation at the time of admission; radiological assessment, including computed tomography (CT) scan, CT angiography (CTA), magnetic resonance imaging (MRI), MR angiography (MRA), and digital subtraction angiography (DSA); additional presence of SAH, intracerebral hematoma (ICH); side and size of aSDH and associated midline shift; aneurysm size and location; case management; outcome according to the Glasgow Outcome Scale (GOS), modified Rankin Score (mRS), and Barthel index (BI).

\section{Results}

The initial search retrieved 85 publications which matched the terms "cerebral aneurysm" AND "subarachnoid hemorrhage" AND "acute subdural hematoma." 59 publications were excluded after screening of titles and abstracts. This left 26 articles potentially eligible for detailed evaluation. Six articles were not included as they did not match the selection criteria. The remaining 20 articles including 82 cases underwent detailed analysis $[2-4,12-14,16-26,28-$ 30]. Characteristics of the 82 cases are summarized in Table 2. Graphs displaying the analyzed data appear in Figure 1.

3.1. Initial Clinical Findings. Most of the patients were admitted with the worst initial clinical SAH grades and with signs of uncal herniation. The distribution according to the WFNS was grade $5(n=46,57.3 \%)$, grade $4(n=$ $14,17.1 \%)$, grade $3(n=6,7.3 \%)$, grade $2(n=8$, $9.8 \%)$, and grade $1(n=8,9.8 \%)$. At admission, signs of uncal herniation, major focal neurological deficits, and minor focal neurological deficits were present in 35 (42.7\%), eight $(9.8 \%)$, and six $(7.3 \%)$ patients, respectively. Fourteen (17.1\%) patients presented in an unstable cardiopulmonary condition (e.g., ventricular arrhythmia, acute heart failure, and sudden pulmonary edema) at the time of admission. Four $(4.9 \%)$ patients died during resuscitation. One (1.2\%) patient was reported to have had prolonged hypoxia.

3.2. Diagnostic Approaches and Radiological Findings. For all patients, the first radiological assessment was a CT scan $(n=$ 82, 100\%). 68 (82.9\%) patients underwent additional DSA, and $11(13.4 \%)$ underwent additional CTA (Figure 2). Four (4.9\%) patients underwent MRA prior to surgery. SAH was detected on initial CT scan in $68(82.9 \%)$ patients. There were $13(15.9 \%)$ cases of pure aSDH without associated SAH. $28(34.1 \%)$ patients presented with additional ICH. In $24(29.3 \%)$ patients, the size of the aSDH was reported (mean \pm SD: $9.6 \pm 3.5$, range: $5-20 \mathrm{~mm}$ ). A total of 30 $(36.6 \%)$ patients were reported as presenting with midline shift associated with aSDH (mean \pm SD: $9.1 \pm 4.0$, range: $4-$ $23 \mathrm{~mm}$ ). All but six cases (7.3\%) of aSDH were documented ipsilateral to the side of the aneurysm. Two cases presented with bilateral aSDH. Aneurysm size was reported in 37 (45.1\%) patients (mean \pm SD: $11.4 \pm 8.1$, range: $1.5-30 \mathrm{~mm}$ ).

3.3. Aneurysm Localization. In most of the cases, the aneurysm was located in the posterior communicating artery (Pcom) $(n=39,46.6 \%)$. The rest of the aneurysms were located in the middle cerebral artery (MCA) $(n=20$, $23.2 \%)$, the anterior communicating artery (Acom) $(n=11$, $13.4 \%)$, the pericallosal artery (Pcal) $(n=8,9.8 \%)$, or the internal carotid artery ICA $(n=4,4.9 \%)$.

3.4. Treatment Strategies. The treatment strategies included urgent hematoma evacuation $(n=59,72 \%)$, surgical aneurysm obliteration in the same procedure as urgent hematoma evacuation $(n=41,50 \%)$, delayed clipping ( $n=$ $10,12.2 \%)$, and delayed coiling $(n=6,7.3 \%)$. Eighteen patients (22\%) died during resuscitation or did not meet the criteria for undergoing any of the invasive procedures due to cardiopulmonary instability. A total of six (7.3\%) patients underwent external ventricular drainage, and ten $(12.2 \%)$ patients were treated with hyperosmolar therapy.

3.5. Outcome. Half of the patients were reported to have favorable outcomes (GOS 5 and GOS 4, $n=39,47.6 \%$ ). Poor 


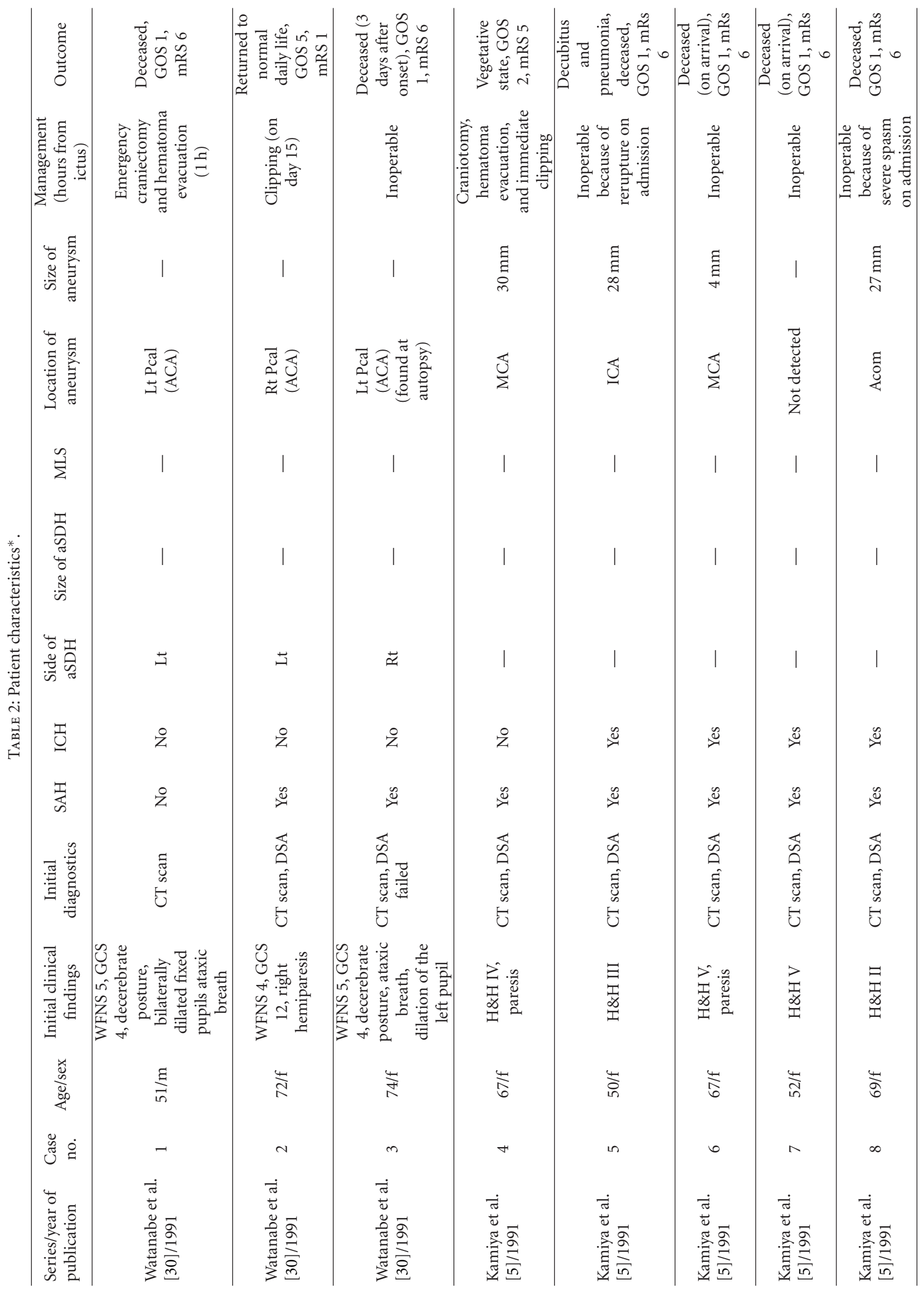




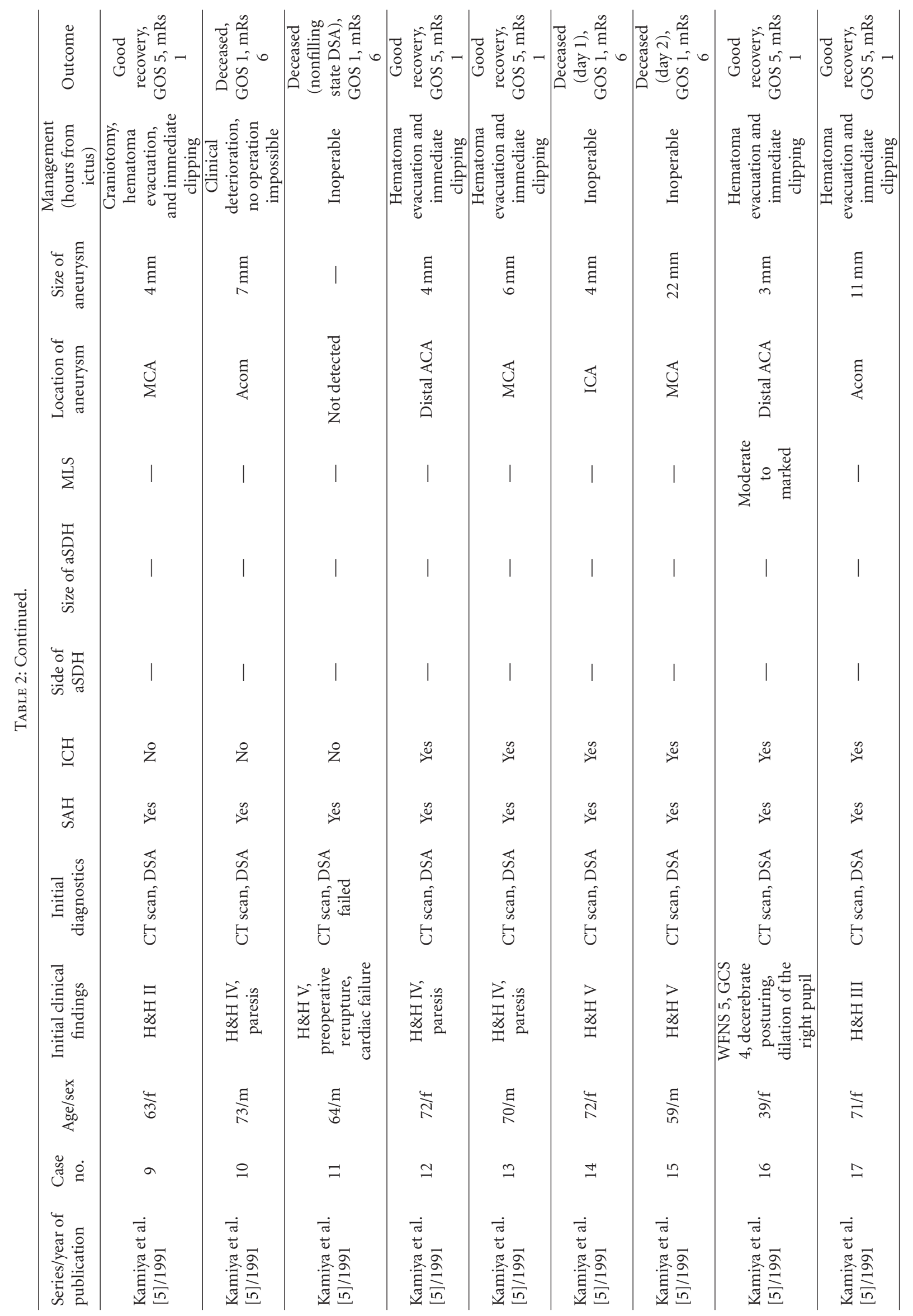




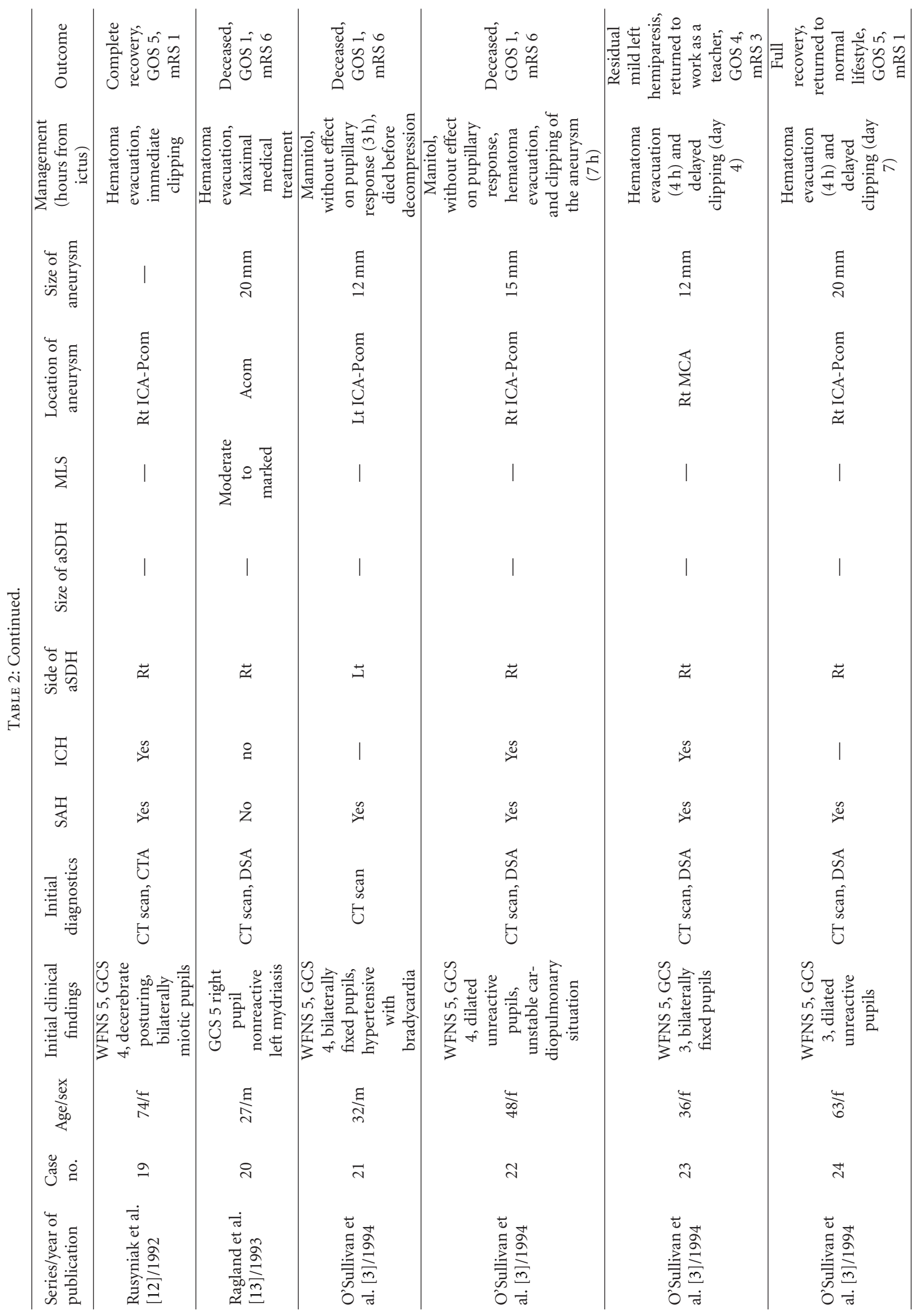




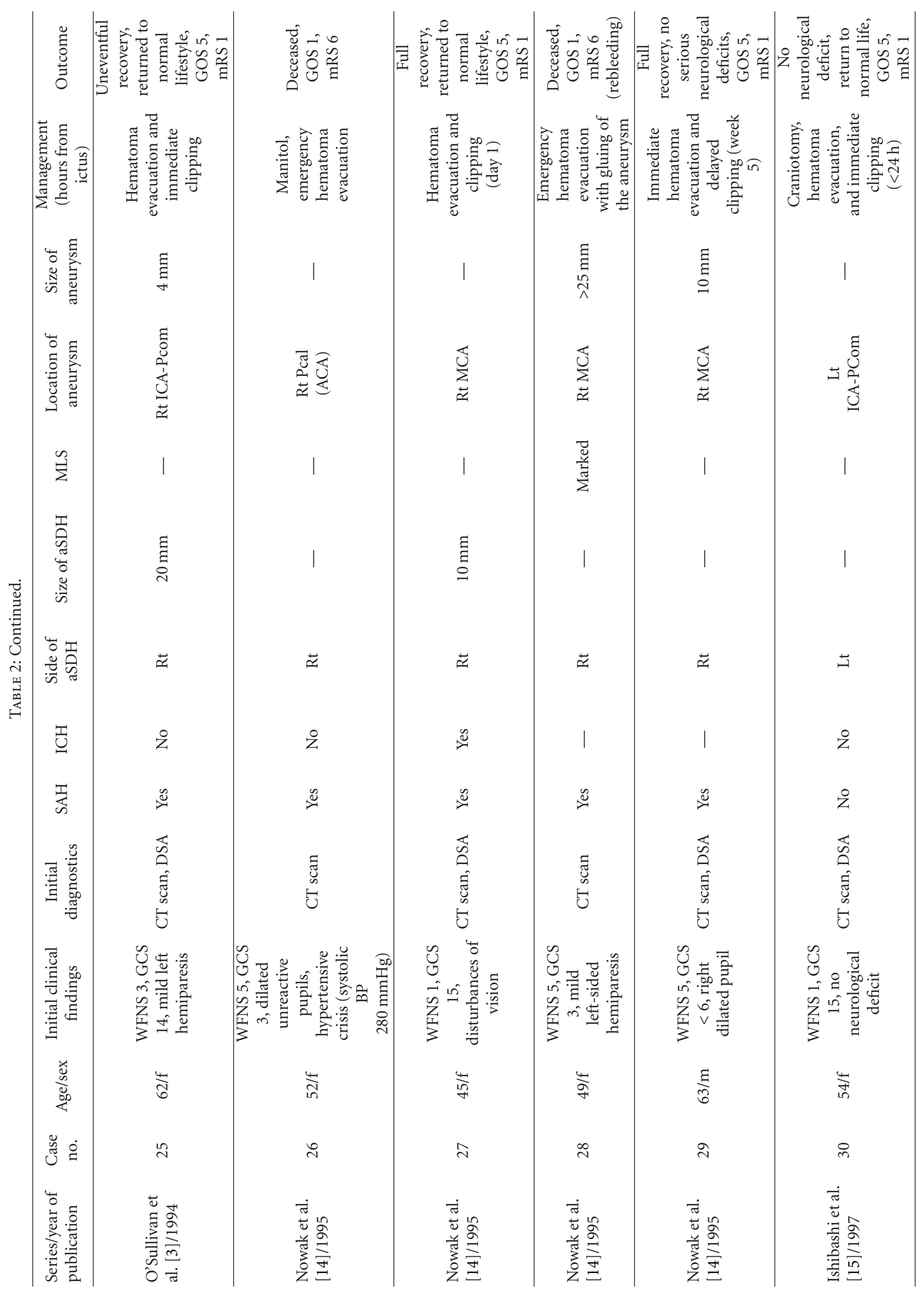




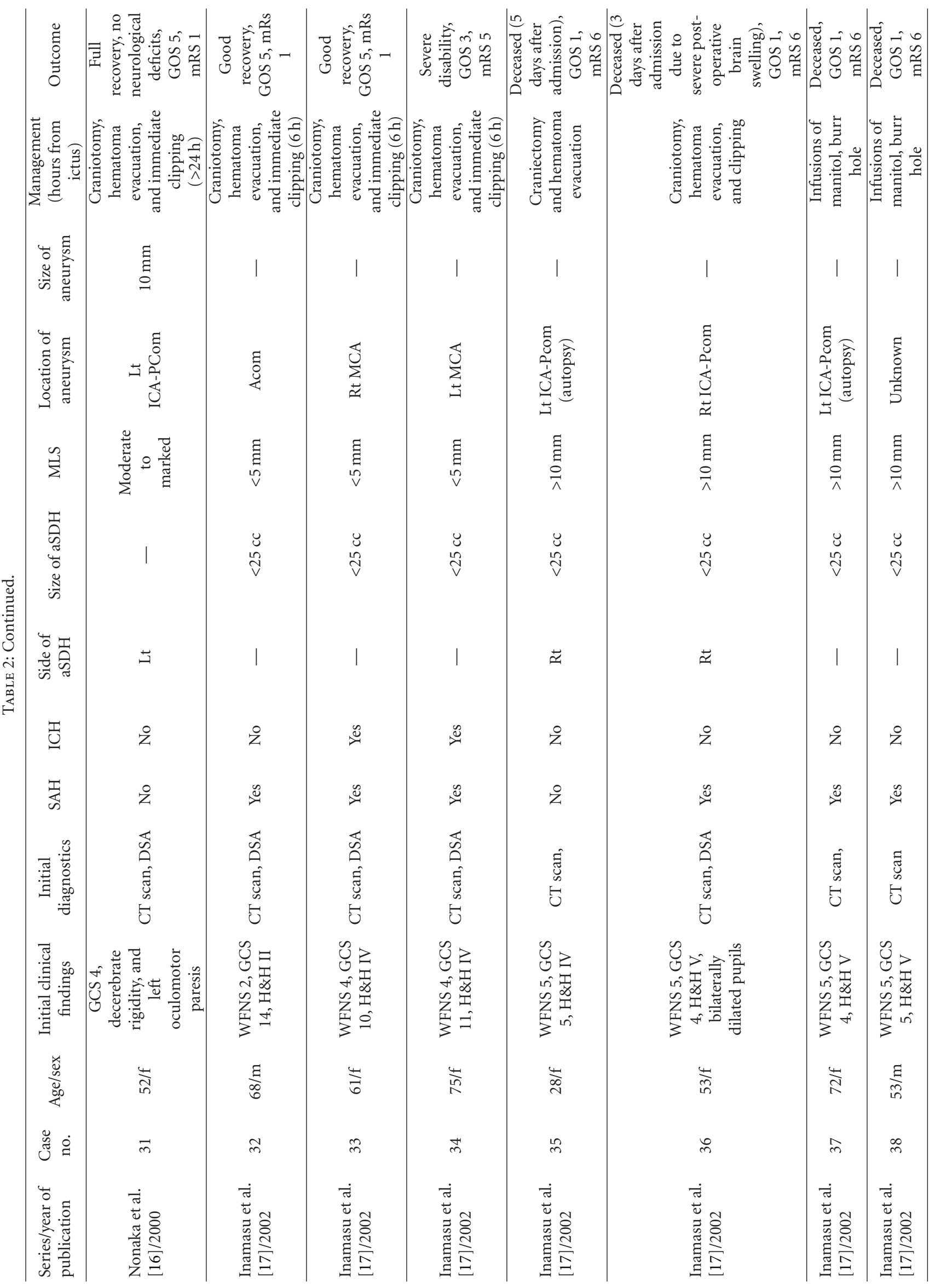




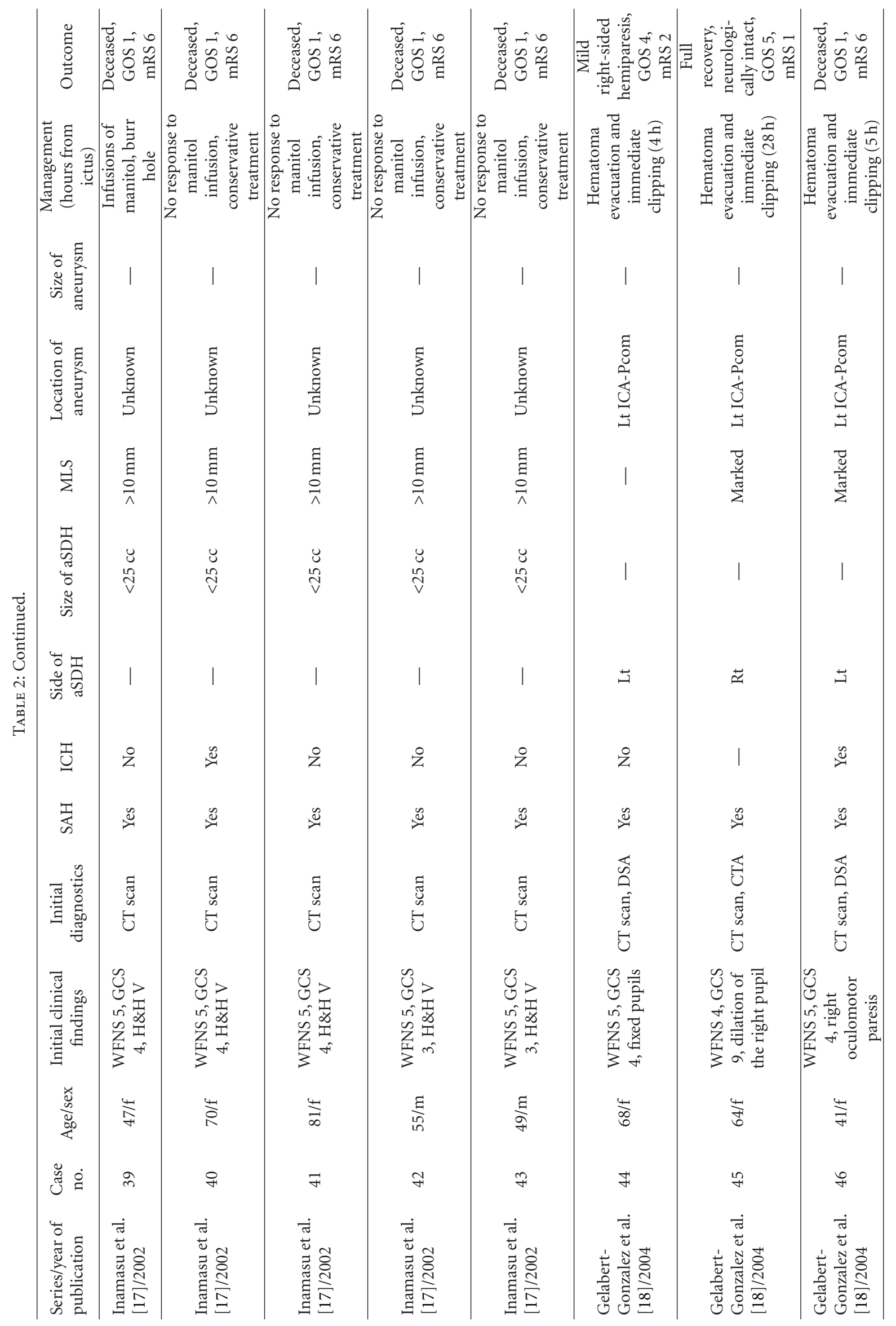




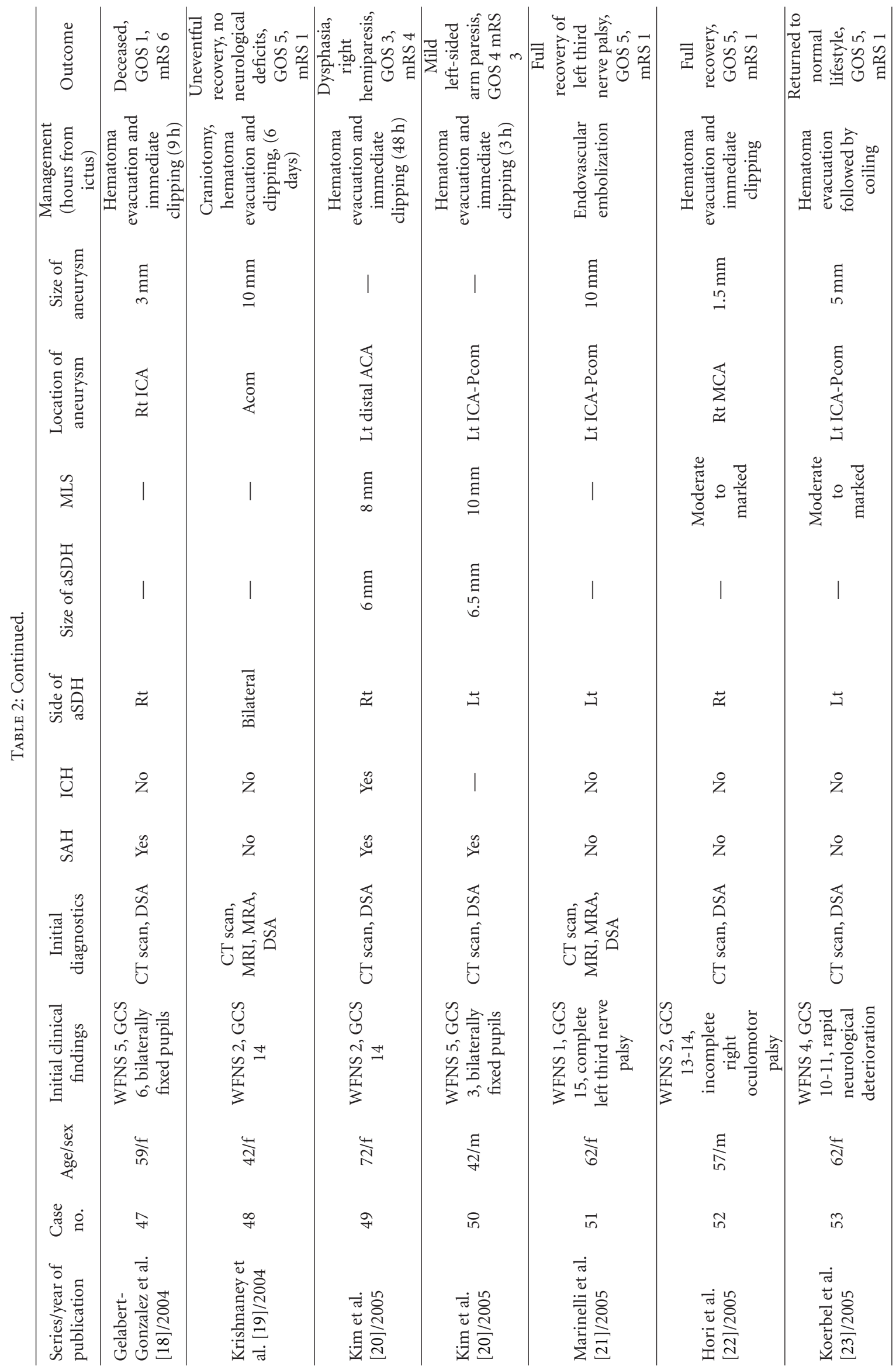




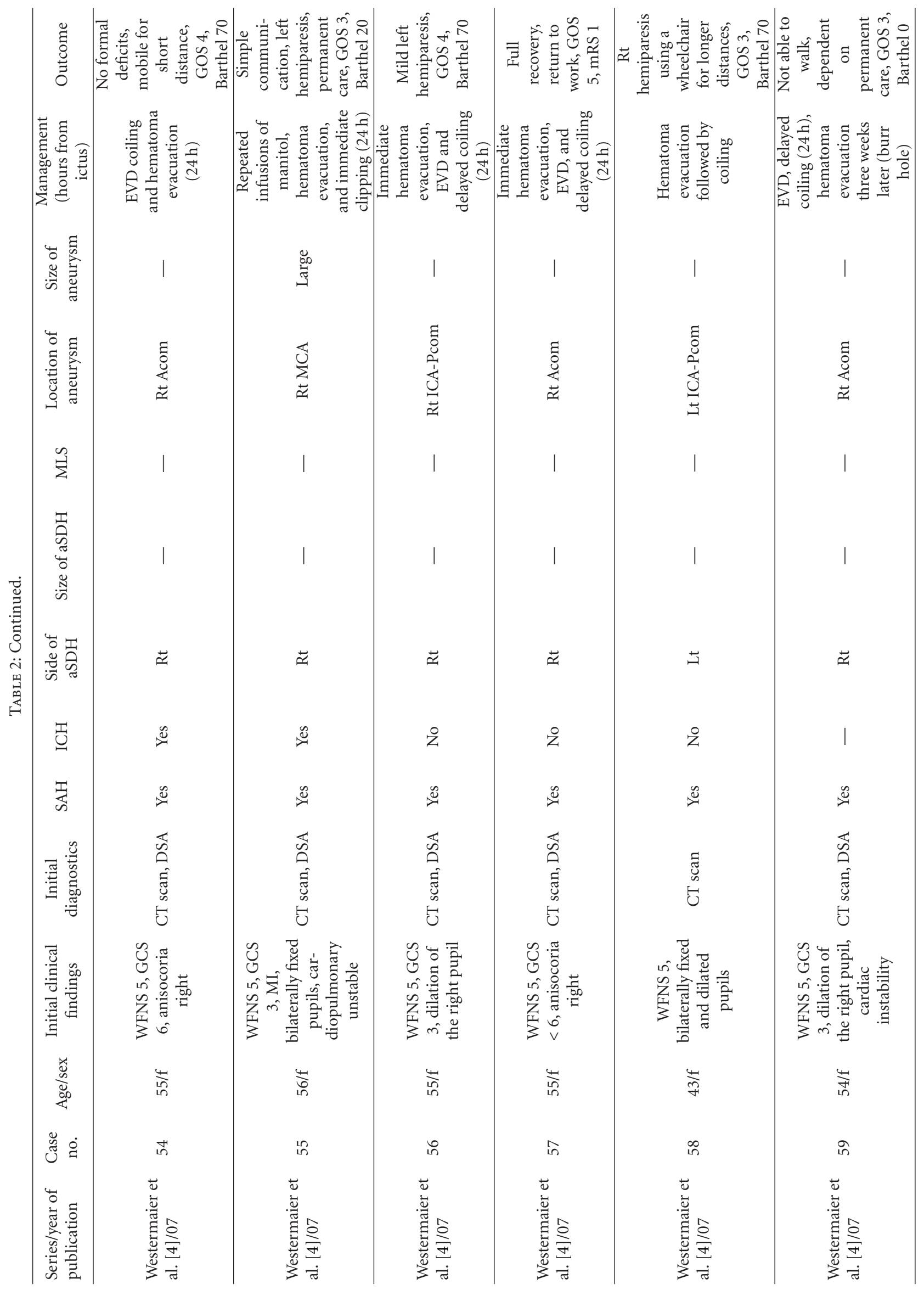




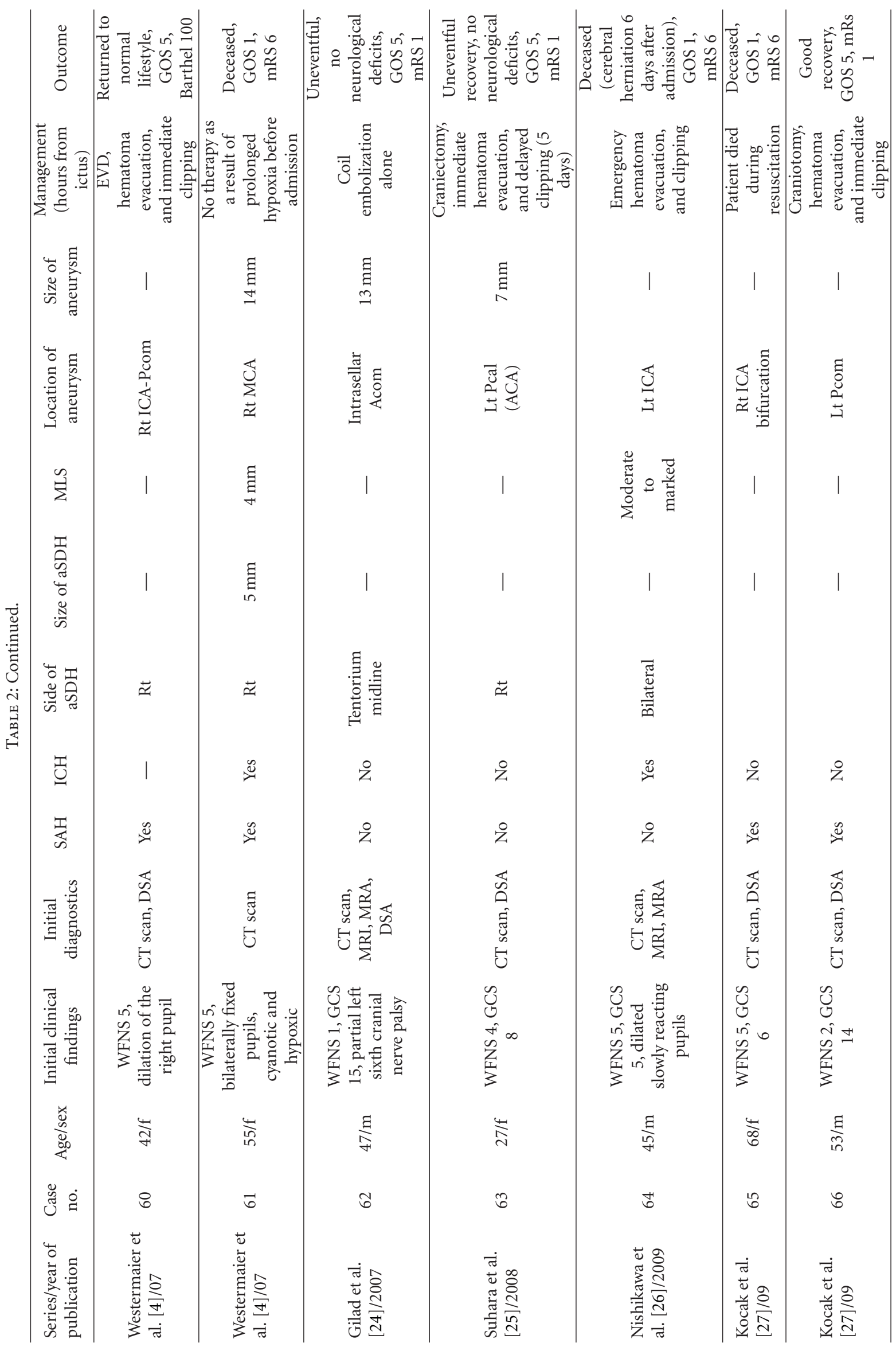




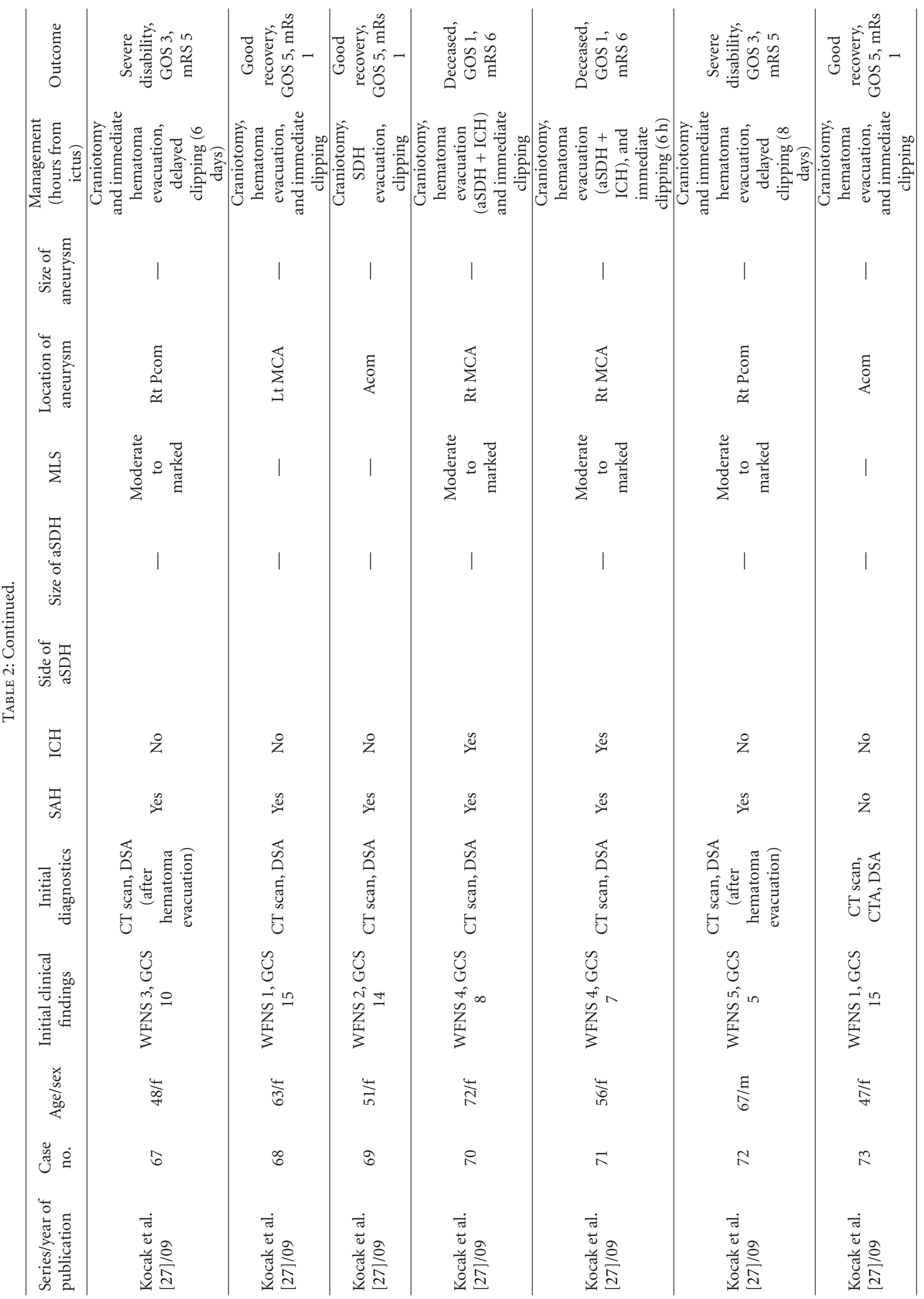




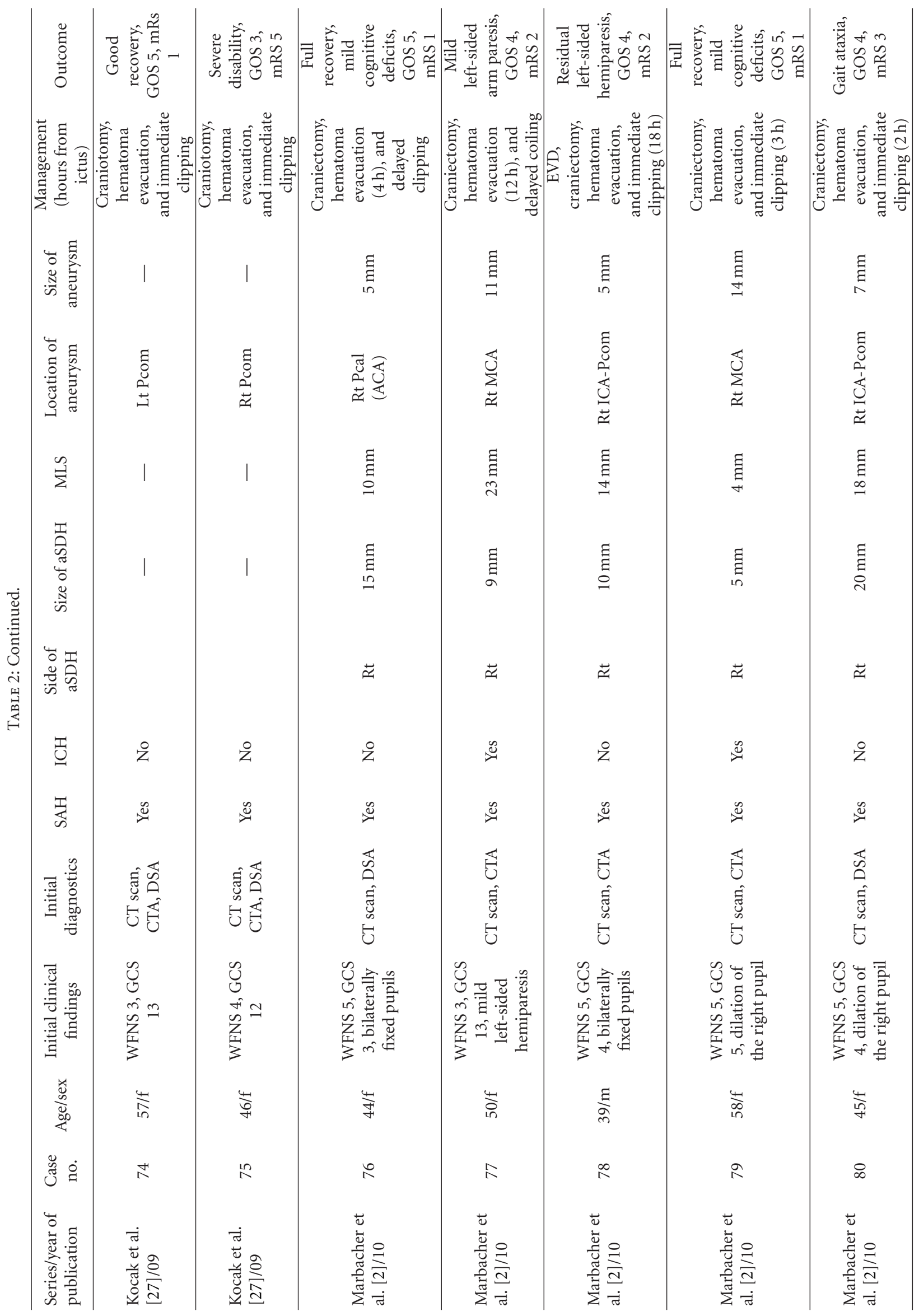




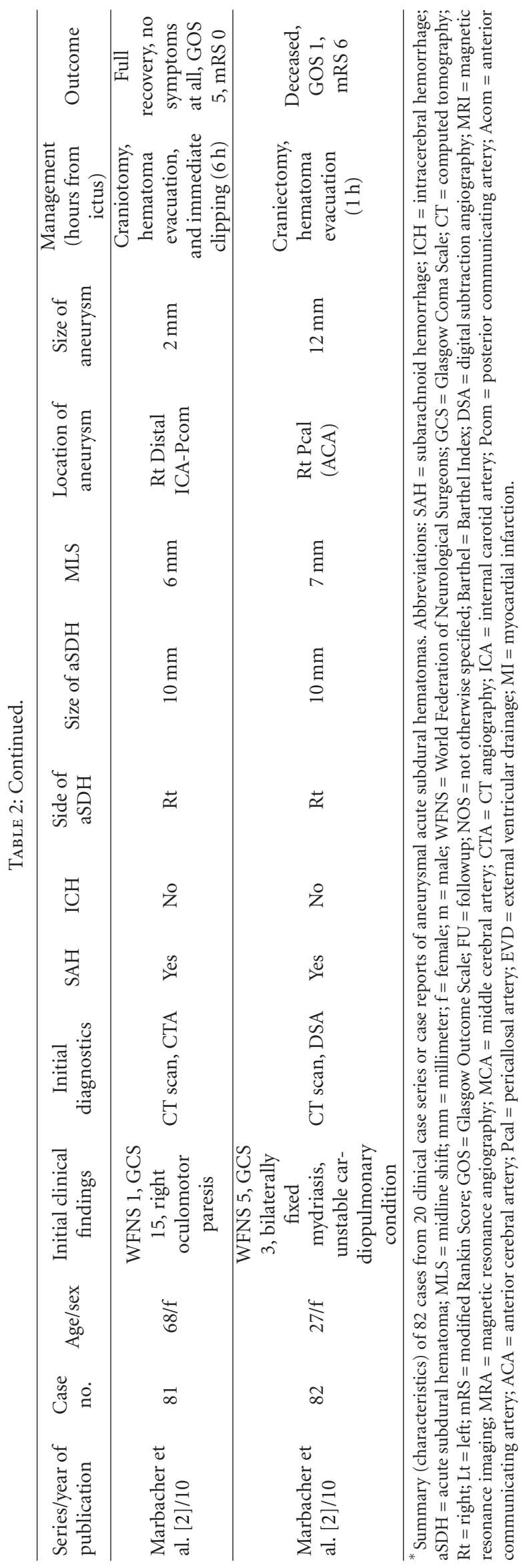




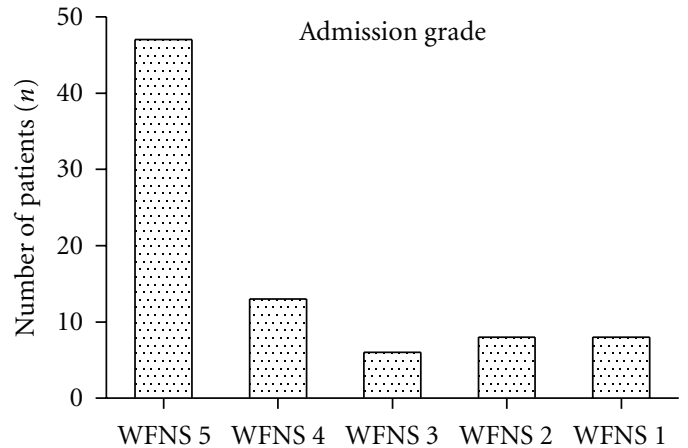

(a)

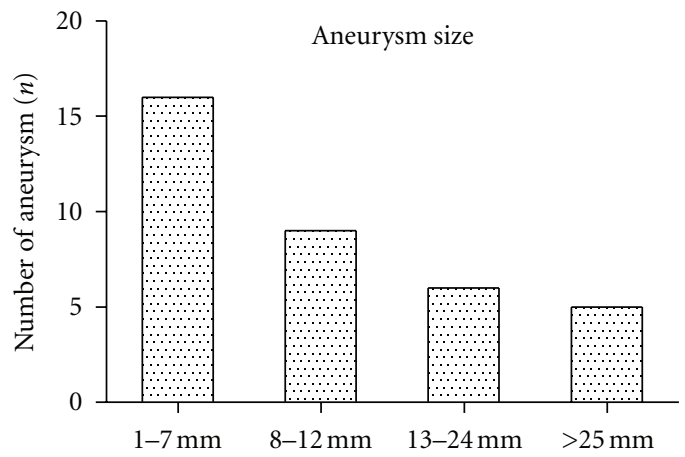

(c)

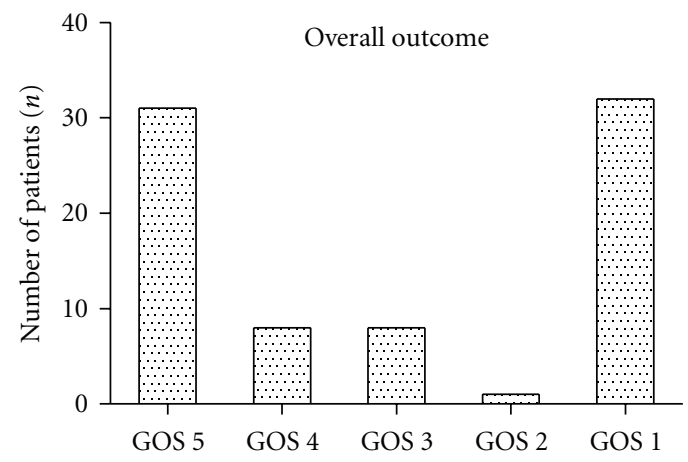

(e)

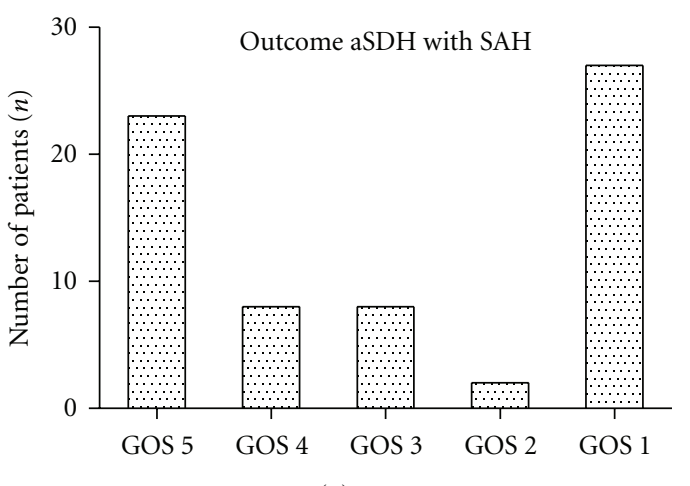

(g)

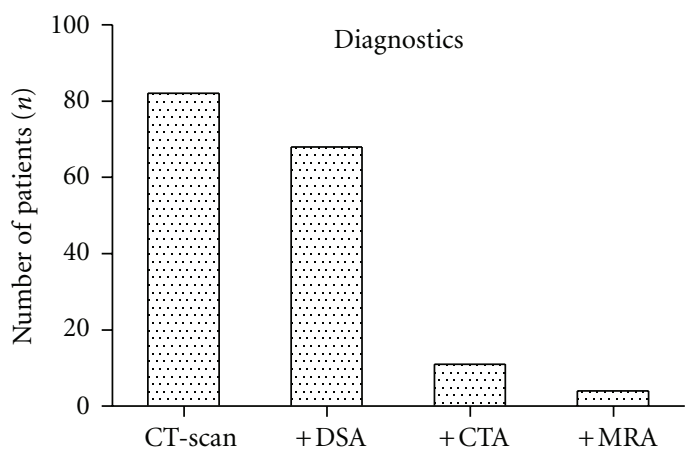

(b)

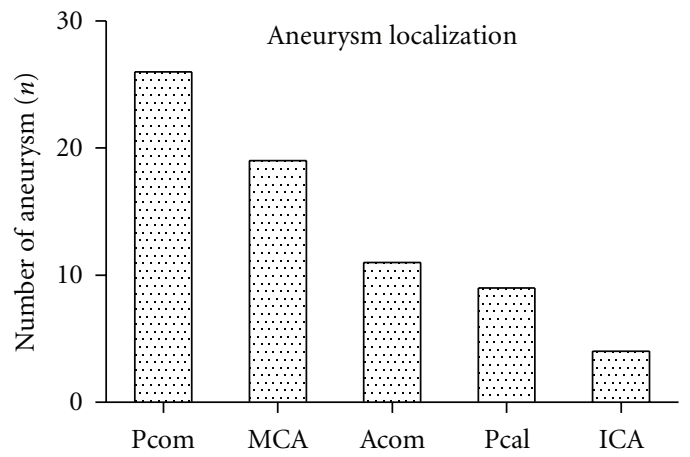

(d)

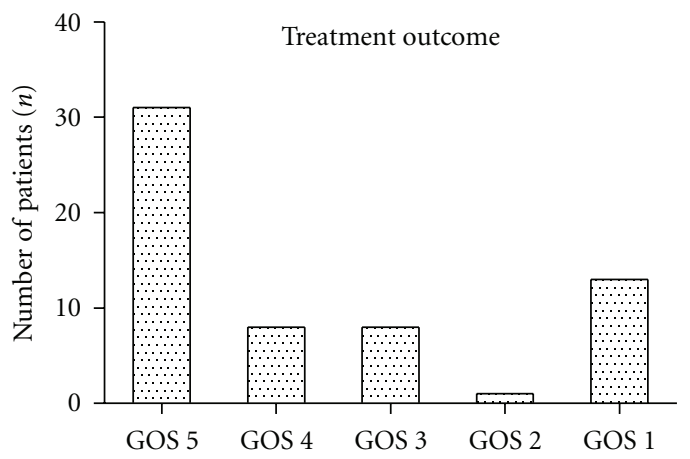

(f)

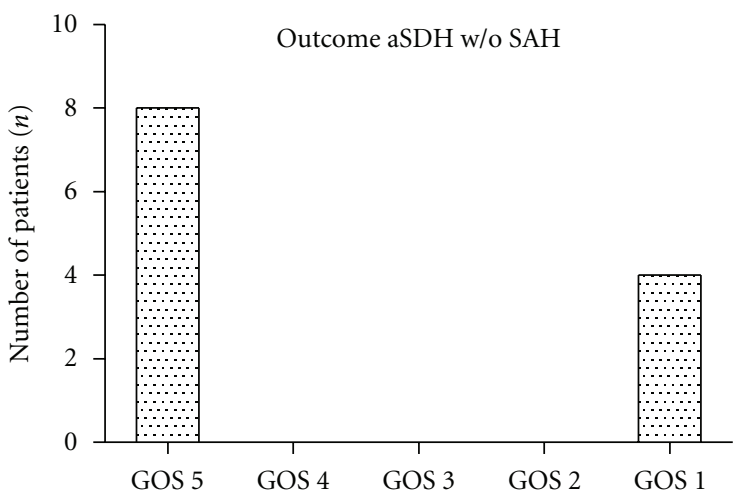

(h)

Figure 1: Data analysis of 82 cases of aneurysmal aSDH $*$. ${ }^{*}$ Abbreviations: WFNS = World Federation of Neurological Surgeons; CT = computed tomography; DSA = digital subtraction angiography; CTA = CT angiography; MRA = Magnetic resonance angiography; mm = millimeter; Pcom = posterior communicating artery; $\mathrm{MCA}=$ middle cerebral artery; Acom = anterior communicating artery; Pcal = pericallosal artery; ICA = internal carotid artery; GOS = Glasgow Outcome Scale. 


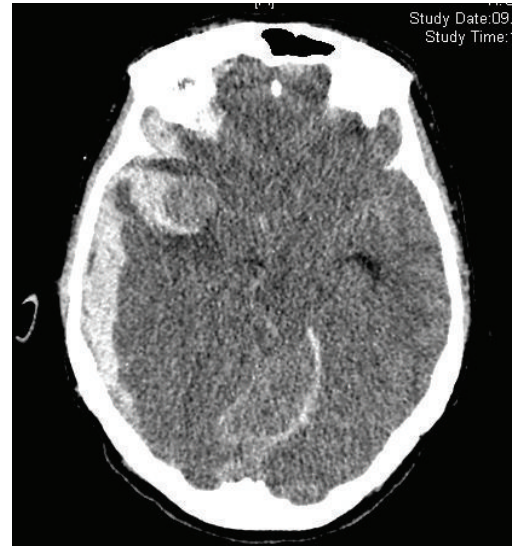

(a)

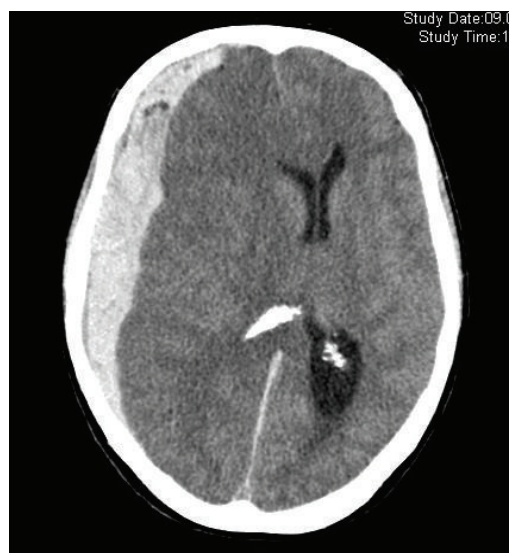

(c)

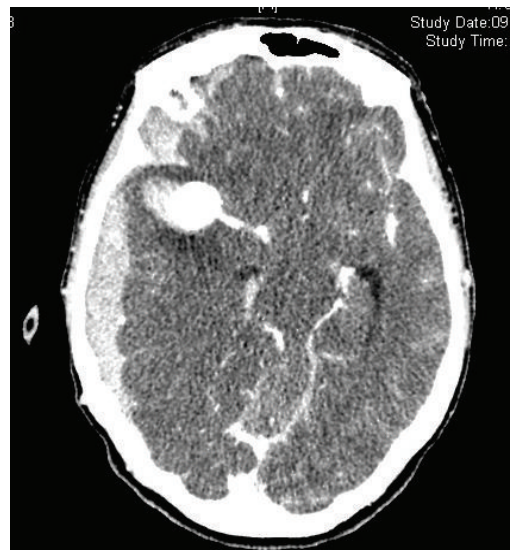

(b)

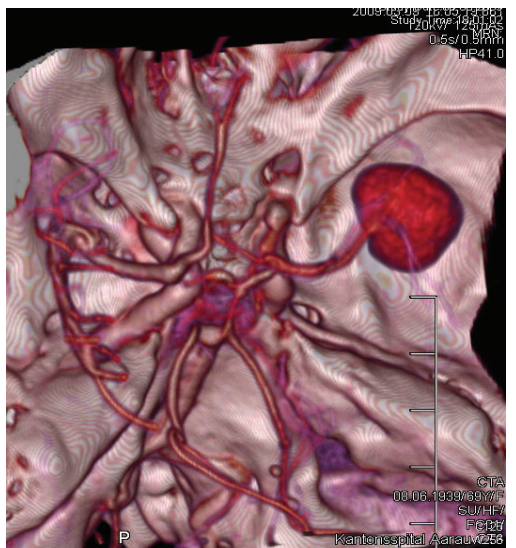

(d)

Figure 2: Illustrative case: Panels (a-d) display axial CT scans with 3D reconstructions showing a right acute subdural hematoma with midline shift after rupture of a giant aneurysm located in the right middle cerebral artery. Panels (a) and (b): noncontrast-enhanced and contrast-enhanced axial CT scan, demonstrating a large aneurysm in the right silvian fissure with surrounding SAH, right-sided aSDH, and uncal herniation. Panel (c) shows a marked midline shift due to the mass effect of the aSDH. Panel (d) depicts the aneurysm with outgoing vessels.

outcome (GOS 3 and GOS 2) was reported in nine (11\%) patients. 32 patients $(26.6 \%)$ had fatal outcomes (GOS 1$)$. Overall distribution according to the GOS was GOS $5(n=$ $31,37.8 \%)$, GOS $4(n=8,9.8 \%)$, GOS $3(n=8,9.8 \%)$, GOS $2(n=1,1.2 \%)$, and GOS $1(n=32,39 \%)$. In $19(23.2 \%)$ out of 32 patients with fatal outcome (GOS 1), the critical status at admission did not allow any surgical or endovascular intervention. Four $(4.9 \%)$ patients died during resuscitation, two $(2.4 \%)$ patients died immediately after diagnosis, and one (1.2\%) patient received no further therapy as a result of prolonged hypoxia before admission. Most of the 63 patients who met the criteria for invasive treatment achieved good outcomes (GOS 5 and GOS 4, $n=$ $39,69.9 \%)$. The distribution of these patients by treatment outcome according to the GOS was GOS $5(n=31,49.2 \%)$, GOS $4(n=8,12.7 \%)$, GOS $3(n=8,12.7 \%)$, GOS $2(n=1,1.6 \%)$, and GOS $1(n=13,20.6 \%)$. Patients who suffered aneurysmal aSDH without SAH demonstrated better outcomes (GOS 5, $n=9,69.2 \%$; GOS $1, n=5$, $38.5 \%$ ) than patients who presented with aneurysmal aSDH and SAH (GOS 5, $n=22,31.4 \%$; GOS $4, n=8,11.4 \%$;
GOS 3, $n=8,11.4 \%$; GOS 2, $n=1,1.4 \%$; GOS 1, $n=27$, $38.6 \%)$.

3.6. Outcome Stratified by Therapeutic Strategies (Table 3). All patients presenting in good clinical condition without rapid neurological deterioration $(n=15)$ demonstrated good outcomes (GOS 5 and GOS 4 ). These outcomes were favorable irrespective of whether hematoma evacuation and aneurysm obliteration were immediate $(n=10)$ or delayed $(n=5)$. However, patients with rapidly deteriorating levels of consciousness (including signs of brain herniation) and urgent $(<24 \mathrm{~h})$ intervention had a higher likelihood of good outcomes (GOS 5 and GOS 4) than patients with rapid deterioration who had undergone delayed $(24 \mathrm{~h})$ treatment (64\% versus $25 \%$ ).

\section{Discussion}

This meta-analysis of 82 reported cases presenting with aneurysmal aSDH and rapid neurological deterioration 


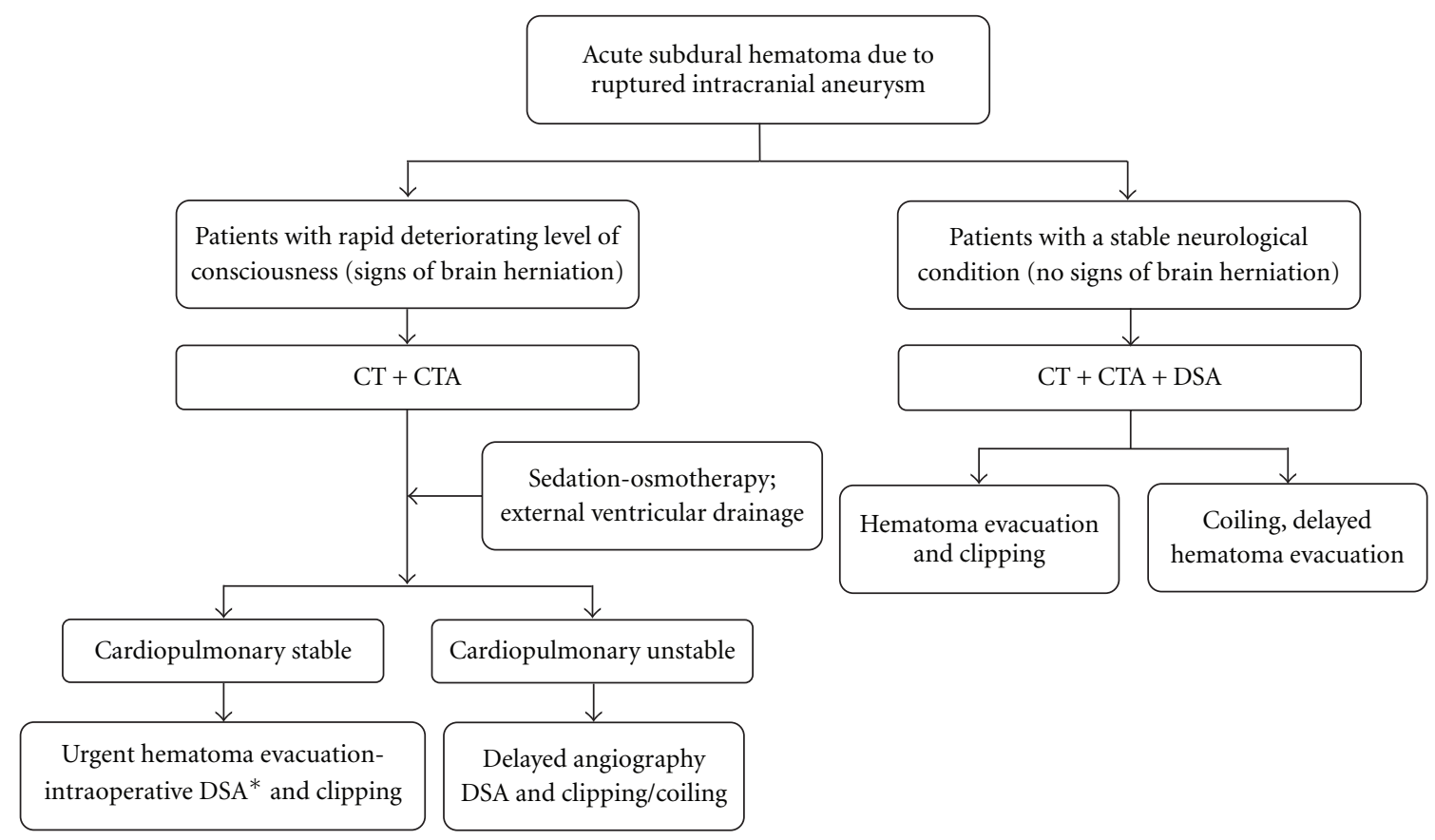

FIGURE 3: Illustrative schematic diagram of the protocol (management algorithm) for diagnosis and treatment of aneurysmal acute subdural hematoma. $\mathrm{CT}=$ computed tomography. CTA $=\mathrm{CT}$ angiography. DSA = digital subtraction angiography. ${ }^{*}=$ if available.

TABLE 3: Outcome stratified according to therapeutic strategies*.

\begin{tabular}{|c|c|c|c|c|c|c|c|}
\hline \multicolumn{4}{|c|}{$\begin{array}{l}\text { Patients presenting with rapidly deteriorating } \\
\text { neurological condition }\end{array}$} & \multicolumn{4}{|c|}{$\begin{array}{l}\text { Patients presenting without rapidly deteriorating } \\
\text { neurological condition }\end{array}$} \\
\hline \multicolumn{2}{|c|}{ Urgent intervention $(<24 \mathrm{~h})$} & \multicolumn{2}{|c|}{ Delayed intervention $(>24 \mathrm{~h})$} & \multicolumn{2}{|c|}{ Urgent intervention $(<24 \mathrm{~h})$} & \multicolumn{2}{|c|}{ Delayed intervention $(>24 \mathrm{~h})$} \\
\hline Outcome & $n(\%)$ & Outcome & $n(\%)$ & Outcome & $n(\%)$ & Outcome & $n(\%)$ \\
\hline GOS $5+4$ & $23(64 \%)$ & GOS $5+4$ & $6(25 \%)$ & GOS $5+4$ & $10(100 \%)$ & GOS $5+4$ & $5(100 \%)$ \\
\hline GOS $3+2$ & $5(14 \%)$ & GOS $3+2$ & $2(8 \%)$ & $\operatorname{GOS} 3+2$ & $0(0 \%)$ & GOS $3+2$ & $0(0 \%)$ \\
\hline GOS 1 & $8(22 \%)$ & GOS 1 & $16(67 \%)$ & GOS 1 & $0(0 \%)$ & GOS 1 & $0(0 \%)$ \\
\hline
\end{tabular}

*Abbreviations: GOS = Glasgow Outcome Scale.

revealed that urgent surgical decompression and immediate occlusion of the aneurysm seem to be an acceptable treatment strategy in order to achieve better outcome (GOS 5 and GOS $4=64 \%$ ). Good outcomes are found in patients maintaining stable neurological condition irrespective of whether intervention was immediate or delayed (GOS $5=$ $100 \%)$. Patients with pure aSDH due to a ruptured aneurysm demonstrated better outcomes than patients who suffered aneurysmal aSDH associated with SAH. Patients in unstable cardiopulmonary condition, with unstable blood pressure and serious ventricular arrhythmias, have the highest risk of unfavorable outcomes. All patients who did not meet the criteria for invasive treatment had fatal outcomes.

Poor clinical presentation per se is not associated with worse outcome. However, the combination of marginal cardiac output and reduced cerebral perfusion and cerebral blood flow due to the mass effect [31] during the acute phase of SAH [32] is likely to result in poor final outcome. Patients presenting in such condition do not meet the criteria for urgent hematoma evacuation, which additionally worsens the likelihood of favorable outcome (GOS 5 and GOS $4=$ $25 \%$ ). Patients in stable hemodynamic condition are suitable for rapid surgical decompression and maximal medical treatment and have a higher chance of recovering in good neurological condition (GOS 5 and GOS $4=64 \%$ ) despite severe SAH and poor initial GCS admission scores. Twothirds of all patients with either poor grade SAH or traumatic aSDH usually do not survive, and functional outcome is rare [33-35]. The good recovery of patients with aneurysmal aSDH might be explained by the space-occupying effect of the hematoma, which mimics a worse clinical situation and does not reflect vital brain destruction.

Pure aSDH due to ruptured intracranial aneurysm is extremely rare. Only 20 cases have been reported so far, including 14 cases during the last two decades [16]. In most cases of aneurysmal aSDH, the history will distinguish a traumatic from a spontaneous cause [1]. However, the absence of hematomas and subarachnoid blood collections related to common aneurysm sites can impede the diagnosis. The finding that pure aneurysmal aSDH results in better 
outcome than aSDH with SAH may be explained by the fact that these patients less frequently have complications (delayed cerebral vasospasm and hydrocephalus).

Due to the rarity of the disease, no guidelines have been established. In most reports, patients have bad clinical features on admission, often presenting in a comatose state with pupillary abnormalities. Fast decision making is mandatory. Determining a differential diagnosis, as well as treatment modalities, can be complicated by the rapid clinical course and the mixture of symptoms due to the ruptured aneurysm or mass effect of the hematoma.

To address the lack of guidelines, we developed a flowchart for treatment of patients with aSDH. However, the evidence for the proposed treatment flowchart comes from case series and case reports with relatively small sample sizes. Therefore, the estimation of effects is imprecise, and clinical recommendations included in the management protocol are weak $[36,37]$.

In patients who are in good neurological condition at the time of admission, management may proceed in a standard manner (Figure 3, left side of the flowchart). After initial CT and CTA examination, DSA is the diagnostic modality of choice to verify the angioarchitecture of the aneurysm. If the aneurysm is suitable for endovascular obliteration and the aSDH remains clinically insignificant, the aneurysm can be occluded during the same procedure [4]. If a decision is made to occlude the aneurysm surgically, DSA provides relevant anatomical information and guidance in determining a clipping strategy and surgical approach.

For the management of patients who are in a coma or whose level of consciousness is deteriorating rapidly, the choice of initial diagnostics is more demanding, and management decisions become difficult (Figure 3, right side of the flowchart). The aSDH may be the major determinant of neurological grade, and prompt hematoma evacuation may be life saving. At the minimum, neuroradiological investigations should consist of an emergency CT and CTA to visualize potential bleeding sources. Emergency treatment modalities such as maximal sedation, osmotherapy, and external ventricular drainage to reverse signs of brain herniation should be performed as quickly as possible. In these cases, the emergency situation forces the neurosurgeon to postpone DSA.

Intraoperative DSA would allow safe and complete aneurysm occlusion to be carried out at the same time as urgent hematoma evacuation $[38,39]$. Patients would be spared a second procedure. However, Westermaier et al. [4] recently presented four patients who underwent separate delayed endovascular coiling after decompression and hematoma evacuation. Despite good neurological recovery in three of these four patients, subjecting patients to two separate procedures rather than clipping at the same time as hematoma removal remains controversial. Patients who present in unstable cardiopulmonary conditions cannot be operated on immediately. It seems that this subgroup of patients is exceptionally at risk of poor outcome. Withholding aggressive therapy in poor-grade patients in order to prevent vegetative survival is highly controversial and cannot be recommended.

\section{Conclusion}

Due to the rarity of aneurysmal aSDH, it remains difficult to define a comprehensive management protocol. In patients with poor neurological grade at admission and rapidly deteriorating levels of consciousness, urgent surgical decompression and immediate aneurysm obliteration result in favorable outcome (GOS 5 and GOS 4; 64\%). Delay of immediate treatment in patients with rapidly deteriorating neurological conditions decreases the likelihood of a favorable outcome (GOS 5 and GOS 4; 25\%). Good outcomes are observed in patients maintaining stable neurological condition irrespective of whether the intervention was immediate or delayed (GOS 5; 100\%). Overall outcome of patients who suffered aneurysmal aSDH without SAH proved to be better (GOS $5,69.2 \%$ ) than the outcome of patients who presented with aneurysmal aSDH and SAH (GOS 5; 31.4\%).

\section{Conflict of Interests}

The authors are solely responsible for the design and conduct of the presented study and report no conflict of interests. No funds were or will be received for this study.

\section{References}

[1] H. Ohkuma, N. Shimamura, S. Fujita, and S. Suzuki, "Acute subdural hematoma caused by aneurysmal rupture: incidence and clinical features," Cerebrovascular Diseases, vol. 16, no. 2, pp. 171-173, 2003.

[2] S. Marbacher, J. Fandino, and A. Lukes, "Acute subdural hematoma from ruptured cerebral aneurysm," Acta Neurochirurgica, vol. 152, no. 3, pp. 501-507, 2010.

[3] M. G. O'Sullivan, M. Whyman, J. W. Steers, I. R. Whittle, and J. D. Miller, "Acute subdural haematoma secondary to ruptured intracranial aneurysm: diagnosis and management," British Journal of Neurosurgery, vol. 8, no. 4, pp. 439-445, 1994.

[4] T. Westermaier, J. Eriskat, E. Kunze, T. Günthner-Lengsfeld, G. H. Vince, and K. Roosen, "Clinical features, treatment, and prognosis of patients with acute subdural hematomas presenting in critical condition," Neurosurgery, vol. 61, no. 3, pp. 482-487, 2007.

[5] K. Kamiya, T. Inagawa, M. Yamamoto, and S. Monden, "Subdural hematoma due to ruptured intracranial aneurysm," Neurologia Medico-Chirurgica, vol. 31, no. 2, pp. 82-86, 1991.

[6] B. Weir, T. Myles, M. Kahn et al., "Management of acute subdural hematomas from aneurysmal rupture," The Canadian Journal of Neurological Sciences, vol. 11, no. 3, pp. 371-376, 1984.

[7] A. Pasqualin, A. Bazzan, P. Cavazzani, R. Scienza, C. Licata, and R. Da Pian, "Intracranial hematomas following aneurysmal rupture: experience with 309 cases," Surgical Neurology, vol. 25, no. 1, pp. 6-17, 1986.

[8] G. Teasdale and B. Jennett, "Assessment of coma and impaired consciousness. A practical scale," The Lancet, vol. 2, no. 7872, pp. 81-84, 1974.

[9] E. H. Botterell, W. M. Lougheed, J. W. Scott, and S. L. Vandewater, "Hypothermia, and interruption of carotid, or carotid and vertebral circulation, in the surgical management of intracranial aneurysms," Journal of Neurosurgery, vol. 13, no. 1, pp. 1-42, 1956. 
[10] W. E. Hunt and R. M. Hess, "Surgical risk as related to time of intervention in the repair of intracranial aneurysms," Journal of Neurosurgery, vol. 28, no. 1, pp. 14-20, 1968.

[11] G. M. Teasdale, C. G. Drake, W. Hunt et al., "A universal subarachnoid hemorrhage scale: report of a committee of the World Federation of Neurosurgical Societies," Journal of Neurology Neurosurgery and Psychiatry, vol. 51, no. 11, p. 1457, 1988.

[12] W. G. Rusyniak, P. C. Peterson, S. H. Okawara, W. H. Pilcher, and E. D. George, "Acute subdural hematoma after aneurysmal rupture; evacuation with aneurysmal clipping after emergent infusion computed tomography: case report," Neurosurgery, vol. 31, no. 1, pp. 129-131, 1992.

[13] R. L. Ragland, N. D. Gelber, H. A. Wilkinson, J. R. Knorr, and A. A. Tran, "Anterior communicating artery aneurysm rupture: an unusual cause of acute subdural hemorrhage," Surgical Neurology, vol. 40, no. 5, pp. 400-402, 1993.

[14] G. Nowak, S. Schwachenwald, U. Kehler, H. Muller, and H. Arnold, "Acute subdural haematoma from ruptured intracranial aneurysms," Acta Neurochirurgica, vol. 136, no. 3-4, pp. 163-167, 1995.

[15] A. Ishibashi, Y. Yokokura, and M. Sakamoto, "Acute subdural hematoma without subarachnoid hemorrhage due to ruptured intracranial aneurysm-case report," Neurologia Medico-Chirurgica, vol. 37, no. 7, pp. 533-537, 1997.

[16] Y. Nonaka, M. Kusumoto, K. Mori, and M. Maeda, "Pure acute subdural haematoma without subarachnoid haemorrhage caused by rupture of internal carotid artery aneurysm," Acta Neurochirurgica, vol. 142, no. 8, pp. 941-944, 2000.

[17] J. Inamasu, R. Saito, Y. Nakamura et al., "Acute subdural hematoma caused by ruptured cerebral aneurysms: diagnostic and therapeutic pitfalls," Resuscitation, vol. 52, no. 1, pp. 7176, 2002.

[18] M. Gelabert-Gonzalez, J. M. Fernandez-Villa, M. Iglesias-Pais, J. Gonzalez-Garcia, and A. Garcia-Allut, "Acute spontaneous subdural haematoma of arterial origin," Neurocirugia, vol. 15, no. 2, pp. 165-170, 2004.

[19] A. A. Krishnaney, P. A. Rasmussen, and T. Masaryk, "Bilateral tentorial subdural hematoma without subarachnoid hemorrhage secondary to anterior communicating artery aneurysm rupture: a case report and review of the literature," American Journal of Neuroradiology, vol. 25, no. 6, pp. 1006-1007, 2004.

[20] J.-M. Kim, J. W. Hur, J.-W. Lee, and M. S. Kim, “Acute subdural hematoma associated with ruptured intracranial aneurysm: diagnosis and emergent aneurysm clipping," Journal of Korean Neurosurgical Society, vol. 37, no. 5, pp. 375-379, 2005.

[21] L. Marinelli, R. C. Parodi, P. Renzetti, and F. Bandini, "Interhemispheric subdural haematoma from ruptured aneurysm: a case report," Journal of Neurology, vol. 252, no. 3, pp. 364-366, 2005.

[22] E. Hori, T. Ogiichi, N. Hayashi, N. Kuwayama, and S. Endo, "Case report: acute subdural hematoma due to angiographically unvisualized ruptured aneurysm," Surgical Neurology, vol. 64, no. 2, pp. 144-146, 2005.

[23] A. Koerbel, U. Ernemann, and D. Freudenstein, "Acute subdural haematoma without subarachnoid haemorrhage caused by rupture of an internal carotid artery bifurcation aneurysm: case report and review of literature," British Journal of Radiology, vol. 78, no. 931, pp. 646-650, 2005.

[24] R. Gilad, G. M. Fatterpekar, D. M. Johnson, and A. B. Patel, "Migrating subdural hematoma without subarachnoid hemorrhage in the case of a patient with a ruptured aneurysm in the intrasellar anterior communicating artery," American Journal of Neuroradiology, vol. 28, no. 10, pp. 2014-2016, 2007.
[25] S. Suhara, A. S. H. Wong, and J. O. L. Wong, "Posttraumatic pericallosal artery aneurysm presenting with subdural haematoma without subarachnoid haemorrhage," British Journal of Neurosurgery, vol. 22, no. 2, pp. 295-297, 2008.

[26] T. Nishikawa, T. Ueba, M. Kajiwara, and K. Yamashita, "Bilateral acute subdural hematomas with intracerebral hemorrhage without subarachnoid hemorrhage, caused by rupture of an internal carotid artery dorsal wall aneurysm. Case report," Neurologia Medico-Chirurgica, vol. 49, no. 4, pp. 152-154, 2009.

[27] A. Kocak, O. Ates, A. Durak, A. Alkan, S. Cayli, and K. Sarac, "Acute subdural hematomas caused by ruptured aneurysms: experience from a single Turkish center," Turkish Neurosurgery, vol. 19, no. 4, pp. 333-337, 2009.

[28] A. Ishibashi and Y. Yokokura, "Clinical analysis of traumatic subarachnoid hemorrhage," Kurume Medical Journal, vol. 38, no. 3, pp. 167-171, 1991.

[29] J. K. Kim and Y. J. Kim, "GDC embolization of intracranial aneurysms with SAH and mass effect by subdural haematoma. A case report and review," Interventional Neuroradiology, vol. 10, no. 1, pp. 47-51, 2004.

[30] K. Watanabe, S. Wakai, S. Okuhata, and M. Nagai, "Ruptured distal anterior cerebral artery aneurysms presenting as acute subdural hematoma-report of three cases," Neurologia Medico-Chirurgica, vol. 31, no. 8, pp. 514-517, 1991.

[31] R. Burger, G. H. Vince, J. Meixensberger, M. Bendszus, and K. Roosen, "Interrelations of laser doppler flowmetry and brain tissue oxygen pressure during ischemia and reperfusion induced by an experimental mass lesion," Journal of Neurotrauma, vol. 16, no. 12, pp. 1149-1164, 1999.

[32] G. A. Schubert, M. Seiz, A. A. Hegewald, J. Manville, and C. Thomé, "Acute hypoperfusion immediately after subarachnoid hemorrhage: a xenon contrast-enhanced CT study," Journal of Neurotrauma, vol. 26, no. 12, pp. 2225-2231, 2009.

[33] J. E. Bailes, R. F. Spetzler, M. N. Hadley, and H. Z. Baldwin, "Management morbidity and mortability of poor-grade aneurysm patients," Journal of Neurosurgery, vol. 72, no. 4, pp. 559-566, 1990.

[34] G. Nowak, R. Schwachenwald, and H. Arnold, "Early management in poor grade aneurysm patients," Acta Neurochirurgica, vol. 126, no. 1, pp. 33-37, 1994.

[35] J. E. Wilberger Jr., M. Harris, and D. L. Diamond, "Acute subdural hematoma: morbidity, mortality, and operative timing," Journal of Neurosurgery, vol. 74, no. 2, pp. 212-218, 1991.

[36] D. Atkins, D. Best, P. A. Briss et al., "Grading quality of evidence and strength of recommendations," British Medical Journal, vol. 328, no. 7454, p. 1490, 2004.

[37] G. H. Guyatt, A. D. Oxman, G. E. Vist et al., "GRADE: an emerging consensus on rating quality of evidence and strength of recommendations," British Medical Journal, vol. 336, no. 7650, pp. 924-926, 2008.

[38] V. L. Chiang, P. Gailloud, K. J. Murphy, D. Rigamonti, and R. J. Tamargo, "Routine intraoperative angiography during aneurysm surgery," Journal of Neurosurgery, vol. 96, no. 6, pp. 988-992, 2002.

[39] G. Tang, C. M. Cawley, J. E. Dion, and D. L. Barow, "Intraoperative angiography during aneurysm surgery: a prospective evaluation of efficacy," Journal of Neurosurgery, vol. 96, no. 6, pp. 993-999, 2002. 


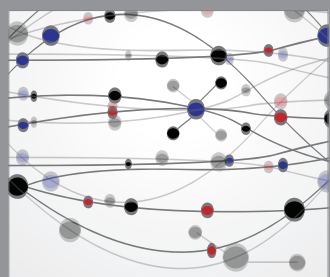

The Scientific World Journal
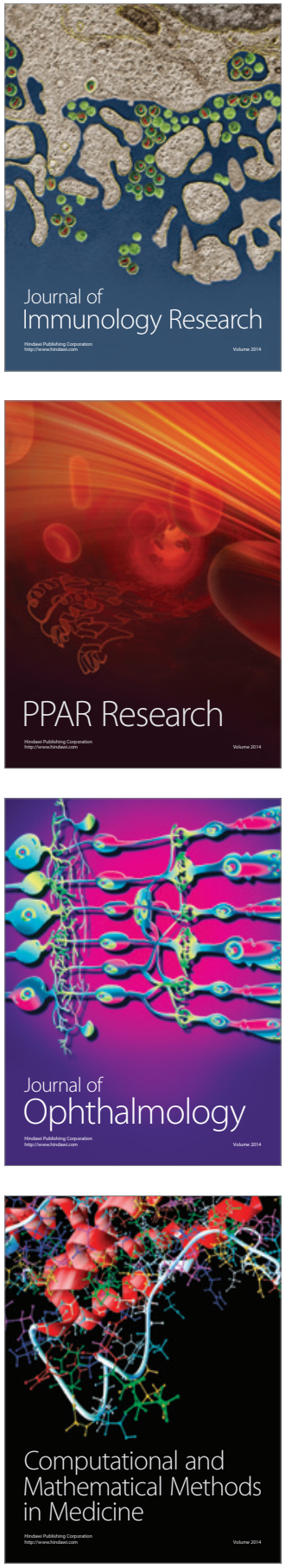

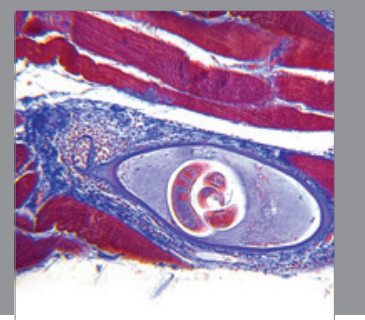

Gastroenterology

Research and Practice
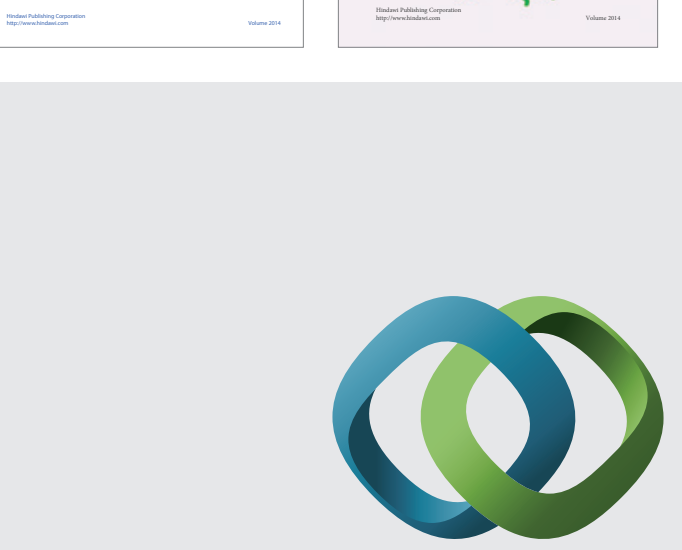

\section{Hindawi}

Submit your manuscripts at

http://www.hindawi.com
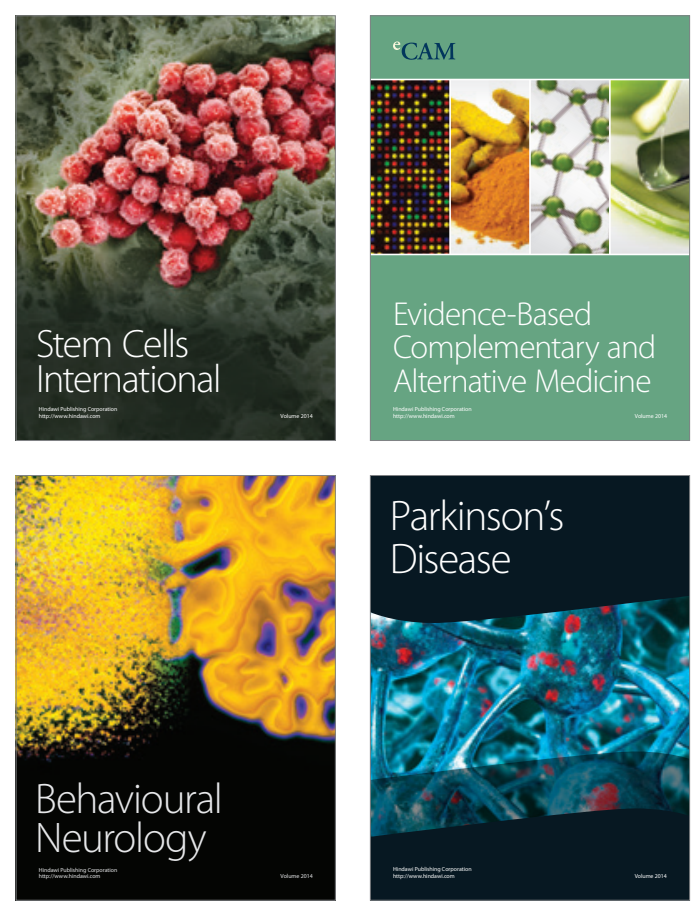

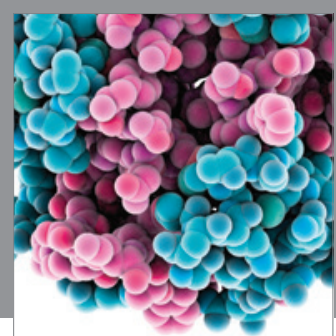

Journal of
Diabetes Research

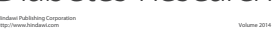

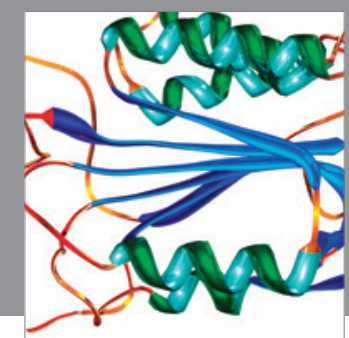

Disease Markers
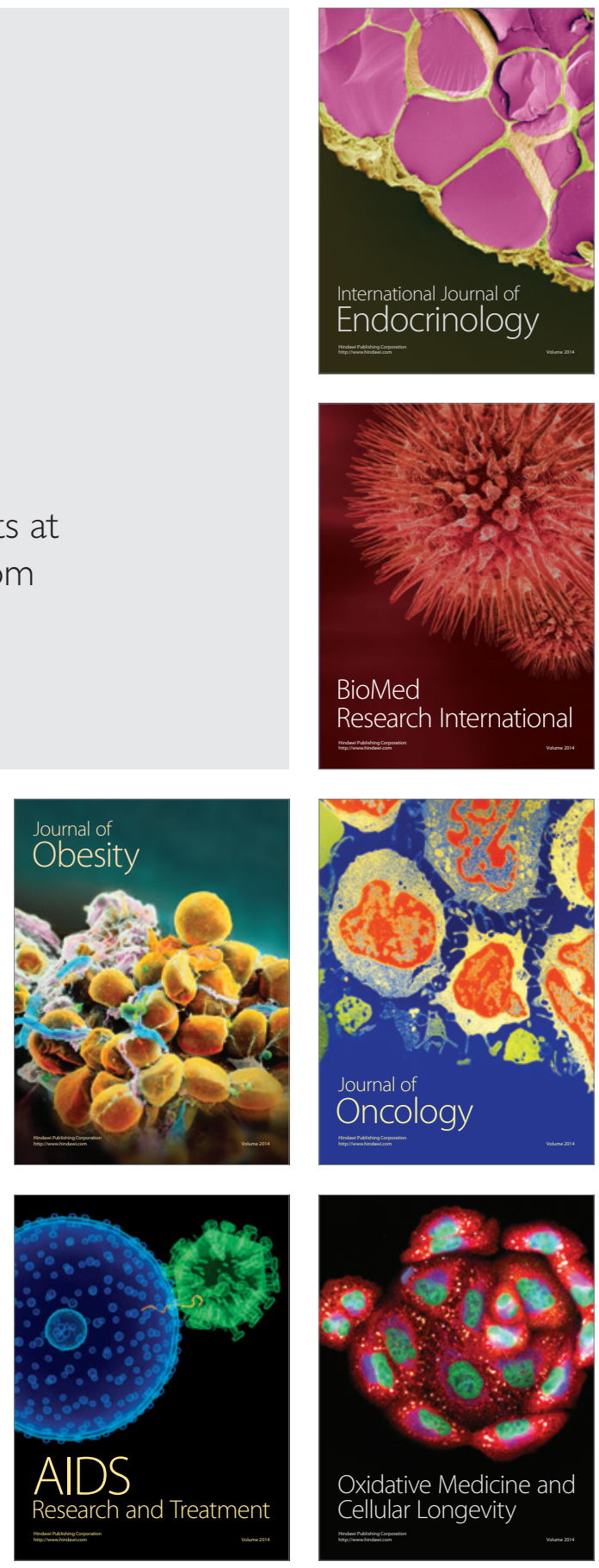